Artículo científico

(Original paper)

\title{
CONTRIBUCIÓN A LA FAMILIA BRACONIDAE (HYMENOPTERA: ICHNEUMONOIDEA) DEL ESTADO DE AGUASCALIENTES, MÉXICO
}

\section{CONTRIBUTION TO THE BRACONIDAE FAMILY (HYMENOPTERA: ICHNEUMONOIDEA) OF THE STATE OF AGUASCALIENTES, MEXICO}

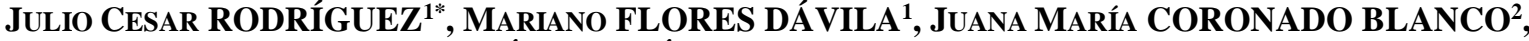 OSWALDO GARCÍA MARTÍNEZ ${ }^{1}$, LUIS ALBERTO AGUIRRE URIBE ${ }^{1}$}
${ }^{1}$ Universidad Autónoma Agraria Antonio Narro, UAAAN, Departamento de Parasitología Agrícola, Calzada Antonio Narro 1923, Buena Vista, 25315, Saltillo, Coahuila.<biol.julio.cesar.rdz@gmail.com>; <marianoflores1920@gmail.com>; <drogarcia@yahoo.com.mx>; <luisaguirreu@yahoo.com.mx>
${ }^{2}$ Facultad de Ingeniería y Ciencias, Universidad Autónoma de Tamaulipas, Centro Universitario Adolfo López Mateos, 87149 Cd. Victoria, Tamaulipas, México. <jmcoronado@docentes.uat.edu.mx>
*Autor de correspondencia: <biol.julio.cesar.rdz@gmail.com>

Recibido: 26/06/2018; aceptado: 24/01/2019; publicado en línea: 29/03/2019

Editor responsable: Arturo Bonet Ceballos

Rodríguez, J. C., Flores-Dávila, M., Coronado-Blanco, J. M., García-Martínez, O., Aguirre-Uribe, L. A. (2019) Contribución a la familia Braconidae (Hymenoptera: Ichneumonoidea) del estado de Aguascalientes. Acta Zoológica Mexicana (nueva serie), 35, 1-10. https://doi.org/10.21829/azm.2019.3502054

RESUMEN. Braconidae es la segunda familia de Hymenoptera con mayor diversidad en el mundo, e incluye parasitoides de insectos y de otros artrópodos. Para Aguascalientes, hasta 2017, sólo se conocían siete subfamilias, ocho géneros y cuatro especies. En el presente trabajo se recolectaron 608 adultos de Braconidae correspondientes a 18 subfamilias, 44 géneros, tres especies y 107 morfoespecies, obtenidos en 111 localidades de los once municipios; de las cuales, 11 subfamilias, 39 géneros y tres especies corresponden a nuevos registros para la entidad. Este trabajo permitió incrementar el registro a 18 subfamilias, 47 géneros y siete especies de Braconidae para el estado de Aguascalientes. La mayoría de los géneros son cosmopolitas y las especies son Neárticas y Neotropicales. Los géneros más abundantes fueron Opius, Aphidius, Trioxys y Aleiodes.

Palabras clave: Taxonomía; biodiversidad; parasitoides

Rodríguez, J. C., Flores-Dávila, M., Coronado-Blanco, J. M., García-Martínez, O., Aguirre-Uribe, L. A. (2019) Contribution to the family Braconidae (Hymenoptera: Ichneumonoidea) of the state of Aguascalientes. Acta Zoológica Mexicana (nueva serie), 35, 1-10. https://doi.org/10.21829/azm.2019.3502054

ABSTRACT. Braconidae is the second most diverse Hymenoptera family in the world, which includes parasitoids of insects and other arthropods. Until 2017, only seven subfamilies, eight genera and four species were known in Aguascalientes. In this research, 608 adults of Braconidae family, corresponding to 18 subfamilies, 44 genera, three species and 107 morphospecies, were obtained in 111 localities of eleven 
municipalities; from those, 11 subfamilies, 39 genera and three species are new records for the state. This work allowed increasing the registry to 18 subfamilies, 47 genera and seven species of Braconidae for the State of Aguascalientes. Most genera are cosmopolitan, and the species are Nearctic and Neotropical. The most abundant genera were Opius, Aphidius, Trioxys and Aleiodes.

Key words: Taxonomy; biodiversity; parasitoids

\section{INTRODUCCIÓN}

El Phylum Arthropoda con más de 1,5 millones de especies descritas contiene el $80 \%$ del total de la fauna mundial, siendo los insectos el grupo más numeroso dentro del Phylum (Zhang, 2011). De éstos, se han descrito de México, hasta el 2009, un total de 47,853 especies, pero se estima que existen cerca de cien mil (Sarukhán et al., 2009). Dentro de la clase Insecta el orden Hymenoptera es uno de los más diversos e incluye muchas especies de importancia económica; el orden a su vez está compuesto por dos subórdenes: Symphyta y Apocrita. En el primero incluye 14 familias. En el segundo la primera serie incluye 35 familias y la segunda cerca de 48, agrupadas en 11 superfamilias que reúnen a las especies de himenópteros parasitoides y las especies formadoras de agallas, además de algunas fitófagas (Delfín \& Chay, 2010).

Dentro de la serie Parasítica, la familia Braconidae constituye una de las más diversas dentro de los insectos; se han descrito cerca de 21,221 especies y se estiman al menos 40,000 en todo el mundo (Yu et al., 2004; Yu et al., 2016). La gran diversidad y las numerosas estrategias de parasitismo, hacen de esta familia un grupo dominante en la regulación de especies (Gaston, 1991; LaSalle \& Gauld, 1993); por tal motivo su estudio se centra en la eficiencia para el control biológico de insectos plaga, el gran potencial como indicador de riqueza y estabilidad de ecosistemas naturales e intervenidos.

En México el estudio de esta familia se ha realizado en todo el país, enfocándose principalmente en tres áreas: 1) el conocimiento de su riqueza taxonómica; 2) investigaciones en ecología, empleando principalmente a estos organismos como bioindicadores y 3) su uso como agentes de control biológico de otros insectos, con aplicaciones potenciales en la agricultura y en actividades forestales (Coronado et al., 2010; Coronado, 2011). Dentro de estas áreas, gran parte de los trabajos pertenecen a taxonomía y sistemática, siendo principalmente estudios realizados en Yucatán, Veracruz, Chiapas, Tamaulipas, Oaxaca, Jalisco y Morelos; en tanto Aguascalientes representa una entidad poco estudiada, teniendo el menor número de registros en el país (Coronado \& Zaldívar, 2014). Los registros de Braconidae para Aguascalientes corresponden a estudios aislados: Escoto-Rocha et al. (2001) registraron a Spathius, Delfín y Wharton (2002) a Aleiodes; y González-Hernández et al. (2003) a Acanthorhogas, Blacus, Cotesia, Cremnops, Peristenus y Vipio. Las especies registradas son Aleiodes atricornis (Cresson), A. molestus (Cresson) (Delfín y Wharton, 2002); Blacus parastigmaticus Sánchez y Wharton (Sánchez et al., 2002) y Vipio moneilemae Gahan (Inayatullah et al., 1998); por tanto, el presente estudio tiene el objetivo de contribuir al conocimiento de las subfamilias, géneros y especies de Braconidae en el estado de Aguascalientes.

\section{MATERIALES Y MÉTODOS}

El estudio se llevó a cabo en diferentes localidades del estado de Aguascalientes, el cual se ubica en la parte central de la República Mexicana, entre los meridianos $101^{\circ} 53^{\prime}$ y $102^{\circ} 52^{\prime}$ de longitud oeste y en los paralelos $22^{\circ} 27^{\prime}$ y $21^{\circ} 28^{\prime}$ de latitud norte, con una superficie de $5,680.33 \mathrm{~km}^{2}$ que representa $0.3 \%$ del total del país. Colinda al norte, este y oeste con el estado de Zacatecas; al sur y este con el de Jalisco. La división política consta de once municipios: Aguascalientes, Asientos, Calvillo, Cosío, Jesús María, Pabellón de Arteaga, Rincón de Romos, San José de Gracia, Tepezalá, San Francisco de los Romo y El 
Llano. Además, el estado cuenta con 13 de los 25 tipos de suelos reconocidos a nivel mundial (Villegas et al., 2008).

Los muestreos para obtener adultos de Braconidae se llevaron a cabo mensualmente utilizando principalmente una red entomológica aérea, dónde por cada localidad (o punto de muestreo) se dieron 100 redazos; esto considerando las altas densidades de vegetación como puntos de muestreo potenciales. Las recolectas se realizaron en el período comprendido de febrero a noviembre de 2017, finalizando con 111 localidades muestreadas (Cuadro 1, Fig. 1).

Cuadro 1. Datos de vegetación y altitud de los sitios de colecta en el Estado de Aguascalientes, México.

\begin{tabular}{llc}
\hline \multicolumn{1}{c}{ Municipio } & \multicolumn{1}{c}{ Vegetación/cultivo } & \multicolumn{1}{c}{$\begin{array}{c}\text { Altitud } \\
(\mathbf{m s n m})\end{array}$} \\
\hline Aguascalientes & Alfalfa, avena, maíz, nogal, malezas. & $1,785-2,036$ \\
Asientos & Alfalfa, durazno, vegetación riparia, matorral, malezas. & $1,977-2,032$ \\
Calvillo & Guayaba, bosques de encinos, malezas. \\
Cosío & Olivos, vides, vegetación riparia, malezas. & $1,656-2,553$ \\
El Llano & $\begin{array}{l}\text { Alfalfa, frutales, maíz, vides, vegetación riparia, bosque de encinos, } \\
\text { malezas. }\end{array}$ & $1,977-1,978$ \\
Jesús María & Alfalfa, maíz, nogal, tomatillo, triticale, malezas. & \\
Pabellón de Arteaga & Alfalfa, ajo, avena, espárragos, maíz, tomate. \\
Rincón de Romos & Alfalfa, chile, frijol, maíz, membrillo, mezquite, moreras, vides, \\
& malezas. & $1,860-2,178$ \\
San Francisco de los & Alfalfa, chile, nogal, malezas. & $1,887-1,927$ \\
Romo & & $1,889-2,010$ \\
San José de Gracia & Bosque de encinos, bosque de pino-encino, vegetación riparia, & $2,037-2,674$ \\
& malezas. & $1,910-2,303$ \\
\hline
\end{tabular}

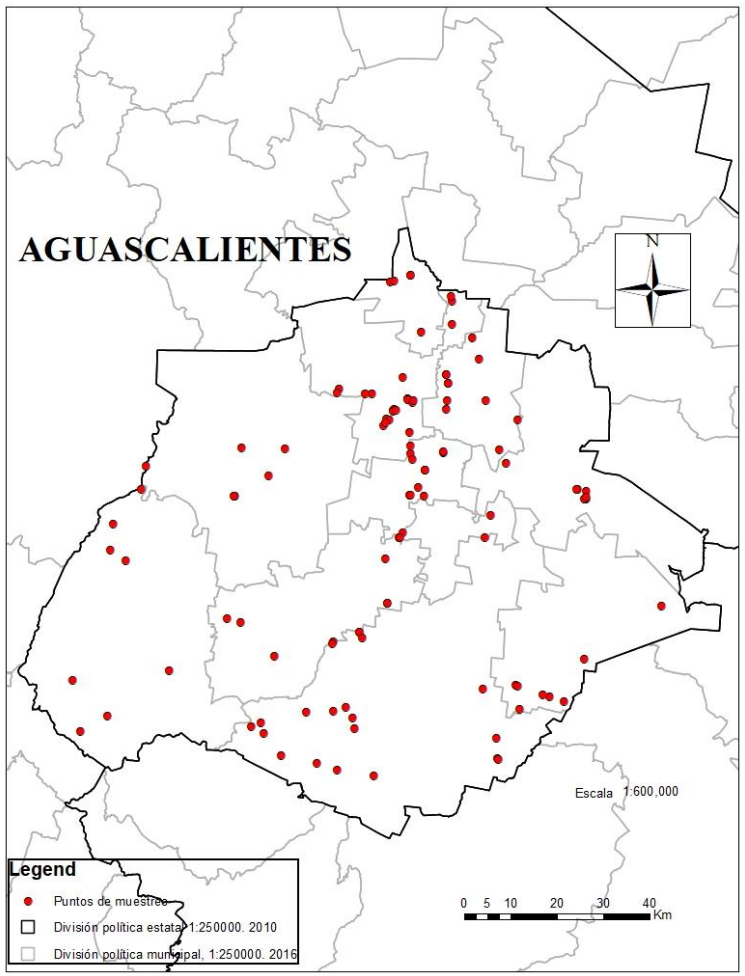

Figura 1. Mapa de Aguascalientes con localidades de muestreo. 
Los especímenes recolectados se colocaron en frascos de 200 c.c. con alcohol etílico al 70\%; posteriormente fueron llevados al Laboratorio de Entomología y Acarología del Departamento de Parasitología Agrícola de la Universidad Autónoma Agraria Antonio Narro (UAAAN), ahí fueron separados los adultos de Braconidae, montados en alfileres entomológicos o puntas de opalina (según el tamaño) y etiquetados con los datos de campo correspondientes.

Los especímenes fueron identificados con el uso del microscopio estereoscópico de la marca Zeiss, siguiendo las claves de Wharton et al. (1998) para identificación a nivel género y las claves de Aguirre et al. (2014, 2015), McIntosh (1855) y Tucker et al. (2015) para las especies determinadas. Se sigue la clasificación según Yu et al. (2016). Los ejemplares fueron depositados en la colección de insectos de la Universidad Autónoma Agraria Antonio Narro (UAAAN), en Buenavista, Coahuila, México.

\section{RESULTADOS}

Se recolectaron 608 especímenes de Braconidae correspondientes a 18 subfamilias, 44 géneros, tres especies y 107 morfoespecies. Con este trabajo, el registro para Aguascalientes quedaría hasta el momento en 18 subfamilias, 47 géneros y siete especies; correspondiendo del presente muestreo 11 subfamilias, 39 géneros y tres especies a nuevos registros para la entidad (Cuadro 2), esto en el periodo abarcado en 2017 y en los once municipios que comprenden la entidad.

Cuadro 2. Braconidae de Aguascalientes, México (febrero-noviembre, 2017).

\begin{tabular}{|c|c|c|}
\hline No. & $\begin{array}{l}\text { Subfamilia } \\
\text { Género }\end{array}$ & Municipio, fecha de colecta (número de especímenes) \\
\hline I. & Acampsohelconinae* & \\
\hline 1. & Urosigalphus* & El Llano: 14-VII (3); Asientos 12-IX (1); Tepezalá:15-X (4). \\
\hline II. & Agathidinae & \\
\hline 2. & Agathis* & $\begin{array}{l}\text { Calvillo: 13-II (1); Aguascalientes 19-IV (1); Pabellón de Arteaga: } \\
\text { 19-IV (1). }\end{array}$ \\
\hline 3. & Bassus** & San Francisco de los Romo: 15-X (1). \\
\hline 4. & Cremnops & \\
\hline 5. & $\begin{array}{l}\text { Cremnops willinki Berta** } \\
\text { Earinus** }\end{array}$ & $\begin{array}{l}\text { Aguascalientes: } 15 \text {-VII (1). } \\
\text { Calvillo: 13-II (1). }\end{array}$ \\
\hline III. & Alysiinae* & \\
\hline 6. & Alysiasta** & El Llano: 12-IX (1). \\
\hline 7. & Aphaereta* & Calvillo:18-VIII (1); San José de Gracia: 08-XI (2). \\
\hline 8. & Chorebus* & $\begin{array}{l}\text { Rincón de Romos: 22-VI (1); Asientos: 12-IX (1); Aguascalientes: } \\
\text { 03-XI (1). }\end{array}$ \\
\hline 9. & Dacnusa* & Aguascalientes: 19-IV (1); San José de Gracia: 18-VIII (1). \\
\hline 10. & Dinotrema** & Calvillo: 13-II (1), 18-VIII (1); San José de Gracia: 08-XI (2). \\
\hline IV. & Aphidiinae* & $\begin{array}{l}\text { Pabellón de Arteaga: 13-II (2), 19-IV (17); San Francisco de los } \\
\text { Romo: 24-III (15), 15-X (2); Jesús María: 24-III (12), }\end{array}$ \\
\hline 11. & Aphidius* & $\begin{array}{l}\text { Aguascalientes: 19-IV (36); Tepezalá: 22-V (8); Rincón de } \\
\text { Romos: 22-V (1), 22-VI (12); El Llano: 14-VII (1); Asientos: 12- } \\
\text { IX (13); Cosío: 15-X (6); San José de Gracia: 08-XI (3). }\end{array}$ \\
\hline 12. & $\begin{array}{l}\text { Diaeretiella* } \\
\text { D. rapae (Mcintosh)** }\end{array}$ & Aguascalientes: 19-IV (2). \\
\hline 13. & Praon* & $\begin{array}{l}\text { Jesús María: 24-III (1); Tepezalá: 22-V (1); Asientos: 12-IX (1); } \\
\text { San José de Gracia: 08-XI (5). }\end{array}$ \\
\hline
\end{tabular}




\begin{tabular}{|c|c|c|}
\hline No. & $\begin{array}{l}\text { Subfamilia } \\
\text { Género } \\
\text { Trioxys** }\end{array}$ & $\begin{array}{l}\text { Municipio, fecha de colecta (número de especímenes) } \\
\text { Pabellón de Arteaga: 13-II (3), 19-IV (13); San Francisco de los } \\
\text { Romo: 24-III (5); Aguascalientes: 19-IV (10), 15-VII (1); } \\
\text { Tepezalá: 22-V (26); Rincón de Romos: 22-V (17), 22-VI (11); El } \\
\text { Llano: 14-VII (2); San José de Gracia: 18-VIII (2), 08-XI (1); } \\
\text { Cosío: 15-X (2). }\end{array}$ \\
\hline $\begin{array}{l}\mathbf{V} . \\
15 .\end{array}$ & $\begin{array}{l}\text { Blacinae } \\
\text { Blacus } \\
\text { B. parastigmaticus Sánchez y } \\
\text { Wharton } \\
\end{array}$ & San José de Gracia: 08-XI (1). \\
\hline $\begin{array}{l}\text { VI. } \\
16 . \\
17 . \\
18 . \\
19 .\end{array}$ & $\begin{array}{l}\text { Brachistinae* } \\
\text { Eubazus }^{*} \\
\text { Nealiolus } * * \\
\text { Schizoprymnus** } \\
\text { Triaspis* }\end{array}$ & $\begin{array}{l}\text { El Llano: 14-VII (1). } \\
\text { Asientos: 12-IX (1). } \\
\text { Calvillo: 18-VIII (1). } \\
\text { San José de Gracia: } 18 \text {-VIII (2). }\end{array}$ \\
\hline VII. & $\begin{array}{l}\text { Vipio } \\
\text { V. moneilemae Gahan }\end{array}$ & $\begin{array}{l}\text { Calvillo: 13-II (1); Pabellón de Arteaga: 13-II (1); Jesús María: 24- } \\
\text { III (2); Aguascalientes: 19-IV (1), 15-VII (1), 03-XI (2); Tepezalá: } \\
\text { 22-V (1), 15-X (1); Cosío: 22-V (1), 15-X (1); El Llano: 14-VII } \\
\text { (2); Jesús María: 18-VIII (2); Asientos: 12-IX (4); San José de } \\
\text { Gracia: 08-XI (3). } \\
\text { Calvillo: 13-II (1); El Llano: 14-VII (1); Rincón de Romos: 13-IX } \\
\text { (2). }\end{array}$ \\
\hline VIII. & $\begin{array}{l}\text { Cheloninae* } \\
\text { Chelonus* }\end{array}$ & $\begin{array}{l}\text { Tepezalá: 22-V (1), 15-X (2); Rincón de Romos: 22-VI (1); El } \\
\text { Llano: 14-VII (1); Aguascalientes: 14-VII (1); Calvillo: 18-VIII } \\
\text { (1); Asientos: 12-IX (5). }\end{array}$ \\
\hline $\begin{array}{l}\text { IX. } \\
23 .\end{array}$ & $\begin{array}{l}\text { Doryctinae } \\
\text { Acanthorhogas }\end{array}$ & \\
\hline $\begin{array}{l}25 . \\
26 .\end{array}$ & $\begin{array}{l}\text { Rhaconotus* } \\
\text { Spathius }\end{array}$ & $\begin{array}{l}\text { Aguascalientes: 19-IV (1), 18-VIII (1), 03-XI (1); Tepezalá: 22-V } \\
\text { (1); El Llano: 14-VII (2); Calvillo: 18-VIII (1); Asientos: 12-IX } \\
\text { (1); Cosío: 15-X (1). } \\
\text { Rincón de Romos: 22-VI (2); El Llano: 14-VII (3); Calvillo: 18- } \\
\text { VIII (1). }\end{array}$ \\
\hline $\begin{array}{l}\mathbf{X .} \\
27 .\end{array}$ & $\begin{array}{l}\text { Euphorinae } \\
\text { Aridelus* }\end{array}$ & \\
\hline $\begin{array}{l}29 . \\
30 .\end{array}$ & $\begin{array}{l}\text { Centistes* } \\
\text { Leiophron* } \\
\text { Meteorus* }\end{array}$ & $\begin{array}{l}\text { San Francisco de los Romo: 24-III (2); Jesús María: 24-III-17 (2); } \\
\text { Aguascalientes: 19-IV (1); Calvillo: 18-VIII (1). } \\
\text { Jesús María: 24-III (11); Aguascalientes: 19-IV (2), 15-VII (2); } \\
\text { Tepezalá: 22-V (1); Rincón de Romos: 22-V (2); 22-VI (2). }\end{array}$ \\
\hline 30. & $\begin{array}{l}\text { Meteorus* } \\
\text { Meteorus arizonensis } \\
\text { Muesebeck** } \\
\text { Microctonus* } \\
\text { Peristenus }\end{array}$ & $\begin{array}{l}\text { Calvillo: 13-II (1); Rincón de Romos: 22-VI (1); Calvillo: 18-VIII } \\
\text { (1). } \\
\text { Jesús María: 24-III (2); Aguascalientes: 19-IV (3); Tepezalá: 22-V } \\
\text { (1), 15-X-17 (1); Rincón de Romos: 22-VI (7); El Llano: 14-VII } \\
\text { (2); Calvillo: 18-VIII (1). }\end{array}$ \\
\hline $\begin{array}{l}\text { XI. } \\
33 .\end{array}$ & $\begin{array}{l}\text { Gnamptodontinae* } \\
\text { Gnamptodon* }\end{array}$ & Calvillo: 18-VIII (1). \\
\hline $\begin{array}{l}\text { XII. } \\
34 .\end{array}$ & $\begin{array}{l}\text { Homolobinae* } \\
\text { Homolobus* }\end{array}$ & Aguascalientes: 19-IV (1); Asientos: 12-IX (3). \\
\hline
\end{tabular}




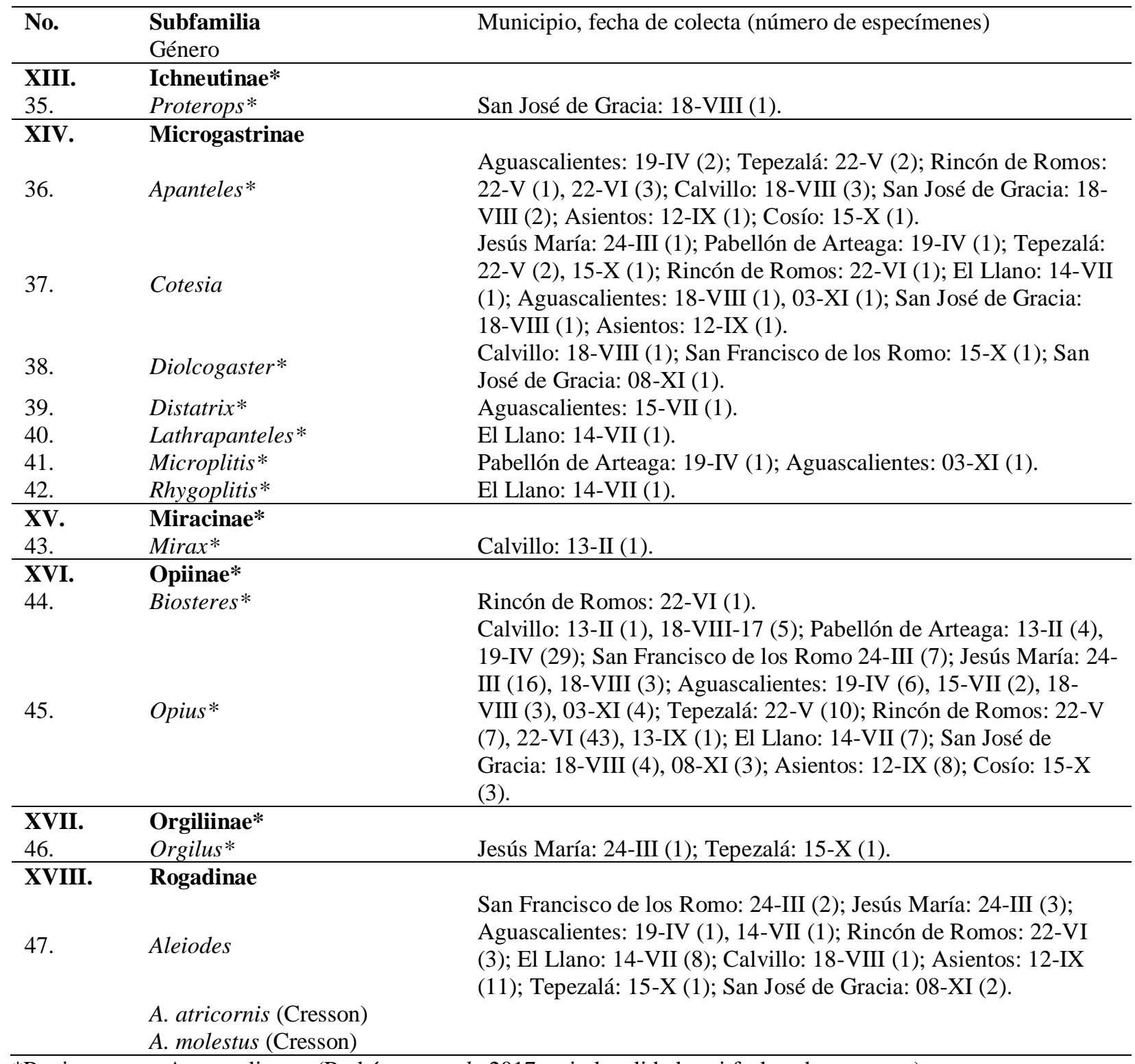

*Registros para Aguascalientes (Rodríguez et al., 2017 - sin localidades ni fechas de muestreo).

**Nuevos registros para Aguascalientes (presente estudio).

Nota: En el listado se anexa a los géneros Acanthorhogas, Spathius (Doryctinae) y Peristenus (Euphorinae) registrados por González et al. (2003) y Escoto et al. (2001) pero no colectados en el presente estudio. Además, se incluyen las cuatro especies registradas con anterioridad para el estado.

Las subfamilias más diversas fueron Microgastrinae, Alysiinae y Euphorinae con siete, cinco y cinco géneros respectivamente, siendo Opiinae y Aphidiinae las más abundantes. Los géneros más abundantes fueron Opius (166) presente en todos los municipios y en todo el período de estudio, Aphidius (128), presente en 10 municipios y en 9 meses, y Trioxys (93) obtenido en ocho municipios y en nueve meses. 
Los especímenes colectados en los municipios arrojaron riqueza genérica distinta para cada uno, siendo los más diversos Calvillo, Aguascalientes, El Llano y San José de Gracia (Cuadro 3). En cuanto a meses de colecta, los meses de julio y agosto fueron los más abundantes en cuanto a riqueza genérica (Cuadro 4).

Cuadro 3. Número de géneros y subfamilias de Braconidae colectados por municipio.

\begin{tabular}{clcc}
\hline No. & Municipio & No. Subfamilias & No. Géneros \\
\hline $\mathbf{1}$ & Aguascalientes & 11 & 20 \\
$\mathbf{2}$ & Asientos & 11 & 13 \\
$\mathbf{3}$ & Calvillo & 12 & 20 \\
$\mathbf{4}$ & Cosío & 5 & 6 \\
$\mathbf{5}$ & El Llano & 11 & 16 \\
$\mathbf{6}$ & Jesús María & 7 & 10 \\
$\mathbf{7}$ & Pabellón de Arteaga & 5 & 7 \\
$\mathbf{8}$ & Rincón de Romos & 9 & 14 \\
$\mathbf{9}$ & San Francisco de los Romo & 6 & 7 \\
$\mathbf{1 0}$ & San José de Gracia & 9 & 15 \\
$\mathbf{1 1}$ & Tepezalá & 10 & 14 \\
\hline
\end{tabular}

\section{DISCUSIÓN}

Hasta antes del 2017, en conjunto, Inayatullah et al. (1998), Escoto et al. (2001), Delfín y Wharton (2002), Sánchez et al. (2002) y González-Hernández et al. (2003) registraron ocho géneros y cuatro especies de siete subfamilias para el estado de Aguascalientes. En el presente estudio se recolectaron los géneros Cremnops, Blacus, Vipio, Cotesia y Aleiodes registrados anteriormente, y no fueron recolectados los géneros Acanthorhogas, Spathius y Peristenus reportados con anterioridad. Rodríguez et al. (2017) registraron un estudio preliminar de los géneros de Braconidae de Aguascalientes, pero no indicaron las localidades ni fechas de colecta de los géneros, los cuales se presentan en este estudio. Con este trabajo más completo, ahora se conocen 18 subfamilias, 47 géneros, siete especies y 107 morfoespecies para el estado de Aguascalientes. Comparando con los registros que dan Coronado y Zaldívar (2014) de las otras entidades, al menos en cuanto a géneros, Aguascalientes dejaría de ocupar el último lugar en registros para colocarse en el sitio 15, superando entidades como Nayarit, Campeche y Puebla, por mencionar algunas. En el rubro de especies, de igual manera dejaría de ocupar el último sitio en cuanto a registros, pero solamente superando al estado de Tlaxcala e igualando en registros a Querétaro y Quintana Roo. La determinación a nivel específico en México es difícil debido a la falta de claves taxonómicas y literatura antigua especializada. Sin embargo, a pesar de esta escasez y a la enorme cantidad de especies descritas mundialmente para el grupo, se encuentran entidades con determinaciones numerosas a este nivel, siendo grupos específicos de subfamilias los más trabajados. Quicke (2012) atribuye que en general el tamaño pequeño de estas avispas complica su determinación, teniendo las especies de cuerpo más grande y más extendidas una mayor probabilidad de ser descritas anteriormente.

Derivado del presente muestreo de colectas de diez meses durante el 2017 y los once municipios, 11 subfamilias, 39 géneros y tres especies corresponden a nuevos registros para la entidad. La posterior colecta en diferentes localidades y con diferentes tipos de muestreo como el uso de platos amarillos, trampas de luz y Malaise será de utilidad para en un futuro tener mejor representatividad de la familia Braconidae en Aguascalientes. 
Cuadro 4. Braconidae de Aguascalientes por mes de muestreo.

\begin{tabular}{clcc}
\hline No. & Mes & No. Subfamilias & No. Géneros \\
\hline $\mathbf{2}$ & Febrero & 7 & 10 \\
$\mathbf{3}$ & Marzo & 7 & 11 \\
$\mathbf{4}$ & Abril & 10 & 16 \\
$\mathbf{5}$ & Mayo & 7 & 11 \\
$\mathbf{6}$ & Junio & 8 & 13 \\
$\mathbf{7}$ & Julio & 11 & 18 \\
$\mathbf{8}$ & Agosto & 12 & 21 \\
$\mathbf{9}$ & Septiembre & 11 & 15 \\
$\mathbf{1 0}$ & Octubre & 11 & 14 \\
$\mathbf{1 1}$ & Noviembre & 8 & 14
\end{tabular}

Los municipios con mayor riqueza genérica presentan diferencias en cuanto a clima y tipo de vegetación con los municipios restantes, lo cual puede influir en la composición de las comunidades de estos parasitoides. En el caso de Calvillo y San José de Gracia se encuentran dentro de uno de los tres tipos generales de grupos climáticos que se presentan en el estado, la zona templada. Esta zona se ubica en la zona montañosa del estado, albergando diferentes tipos de bosques de encino o bosques mixtos (encinoconíferas) (Siqueiros et al., 2016). Calvillo, por su extensión territorial, abarca otra de las mencionadas zonas, la tropical, cubierta por matorrales subtropicales secundarios que han sustituido a las comunidades prístinas de selva baja caducifolia, la cual queda como relicto en algunas zonas conservadas (Siqueiros et al., 2016). Los municipios restantes ocupan la zona árida, la cual se encuentra cubierta por vegetación xerofítica.

Julio y agosto fueron los meses con mayor abundancia en cuanto a riqueza genérica. Si se considera que el clima predominante en la región es seco semicálido (INEGI, 1996), y que las temperaturas mensuales más altas y mayores precipitaciones acumuladas se dan en los meses de agosto y septiembre $\left(29\right.$ a $35^{\circ} \mathrm{C} \mathrm{y}$ de 50 a $60 \mathrm{~mm}$, respectivamente), se puede correlacionar con la diversidad presente. El hecho de que desde el mes de julio comenzó el aumento en la riqueza, probablemente se deba al aumento de temperatura que causa el cambio climático, puesto que es uno de los factores ambientales más importantes que tiene influencia directa sobre la distribución, desarrollo, sobrevivencia y reproducción de los insectos (AlonsoHernández et al., 2014).

Respecto a las relaciones zoogeográficas de los géneros colectados en Aguascalientes, el mayor componente es el Cosmopolita. De los 44 géneros determinados, 43 tienen presencia en las dos zonas zoogeográficas presentes en el continente, siendo Schizoprymnus el género que se presenta únicamente en la región Neártica. Al comparar la cantidad de especies registradas para cada región, 30 géneros presentan una mayor cantidad de especies en la zona Neártica, los 14 restantes tienen mayor cantidad de especies en la zona Neotropical (basados en Yu et al., 2016). Este tipo de relaciones a nivel género es el que se presenta en la parte Neártica de México como es el estado estudiado.

Por su parte, las especies determinadas Diaeretiella rapae (M'Intosh, 1855) y Meteorus arizonensis Muesebeck, 1923 son Neárticas y Neotropicales, mientras que Cremnops willinki Berta, 1998 ha sido registrada solamente en la zona Neotropical (Yu et al., 2016).

\section{CONCLUSIONES}

El estudio de la familia Braconidae en México se había concentrado en pocos estados y tipos de vegetación. En los últimos años, las investigaciones se han extendido a otras entidades como es el caso del estado de Aguascalientes en este estudio, donde la mayoría de las subfamilias y la gran mayoría de los géneros 
obtenidos constituyen nuevos registros para la entidad. Los meses con mayor número de bracónidos colectados fueron julio, agosto y septiembre. Las subfamilias con más géneros fueron Microgastrinae, Alysiinae y Euphorinae, en contraste Opiinae y Aphidiinae fueron las más abundantes en cuanto a especímenes.

Así pues, para el presente trabajo se recolectaron 608 especímenes de Braconidae agrupados en 18 subfamilias, 44 géneros, tres especies y 107 morfoespecies; de las cuales 11 subfamilias, 39 géneros y tres especies corresponden a nuevos registros para la entidad. Con esto, se tiene evidencia de la riqueza de estas avispas en el estado, por tanto, se recomienda seguir con los trabajos, sobre todo en áreas no muestreadas.

AgRAdeCIMIEnTOS. A la UAAAN, por el apoyo para la realización de este proyecto. A los revisores anónimos por su contribución a la mejora del presente artículo.

\section{LITERATURA CITADA}

Aguirre, H., Shaw, S. R. (2014) Neotropical species of Meteorus Haliday (Hymenoptera: Braconidae: Meteorinae) parasitizing Arctiinae (Lepidoptera: Noctuoidea: Erebidae). Zootaxa, 3779, 353-367. http://dx.doi.org/10.11646/zootaxa.3779.3.3.

Aguirre, H., de Almeida, L. F., Shaw, S. R., Sarmiento, C. E. (2015) An illustrated key to Neotropical species of the genus Meteorus Haliday (Hymenoptera, Braconidae, Euphorinae). ZooKeys, 489, 33 94.

http://dx.doi.org/10.3897/zookeys.489.9258

Alonso-Hernández, N., Sánchez-García, J. A., Figueroa de la Rosa, J. I., López-Martínez, V., Martínez-Martínez, L., Pérez-Pacheco, R., Granados-Echegoyen, C. (2014) Distribución espacial de bracónidos (Hymenoptera) reportados en el estado de Oaxaca. Acta Zoológica Mexicana, 30, 564-594.

Coronado-Blanco, J. M. (2011) Braconidae (Hymenoptera) de Tamaulipas, México. Editorial Planeta. México, 203 pp.

Coronado-Blanco, J. M., Zaldívar-Riverón, A. (2014) Biodiversidad de Braconidae (Hymenoptera: Ichneumonoidea) en México. Revista mexicana de biodiversidad, 85, 372-378. http://dx.doi.org/10.7550/rmb.32000

Coronado-Blanco, J. M., Ruíz-Cancino, E., López-Martínez, V., Sánchez-García, J. A., Figueroa-De La Rosa, J. I., Delf́in- González, H. (2010) Braconidae (Hymenoptera) en México. Pp. 80-87. In: Toledo-Hernández, V. H., Corona-López, A. M., Flores-Palacios, A., Tovar-Sánchez, E., Coronado-Blanco, J. M., Ruíz-Cancino, E. (Eds). Memoria II Taller Internacional de Recursos Naturales (CD), Cuernavaca, Morelos, México.

Delfín-González, H., Chay-Hernández, D. (2010) Riqueza de Hymenoptera. Pp. 239-240. In: Durán, R., Méndez, M. (Eds). Biodiversidad y Desarrollo Humano en Yucatán. CICY, PPD-FMAM, CONABIO, SEDUMA, Yucatán, México.

Delfín-González, H., Wharton, R. A. (2002) Distribution of species and species-groups of Aleiodes (Hymenoptera: Braconidae) in Mexico. Folia Entomológica Mexicana, 41, $215-227$.

Escoto-Rocha, J., Cruz-Gutiérrez, H. J., Delgado-Saldívar, L. (2001). Biodiversidad de himenópteros del Estado de Aguascalientes. Investigación y Ciencia-Universidad Autónoma de Aguascalientes, 9, 20-27.

Gaston, K. J. (1991) The magnitude of global insect species richness. Conservation Biology, 5, 283-296. https://doi.org/10.1111/j.1523-1739.1991.tb00140.x

González-Hernández, A., Wharton, R. A., Sánchez-García, J. A., López-Martínez, V., Lomelí-Flores, J. R., Figueroa-De La Rosa, J. I., Delfín González, H. (2003) Catálogo ilustrado de Braconidae 
(Hymenoptera: Ichneumonoidea) de México. Universidad Autónoma de Nuevo León- CONABIO - CONACyT. CD Interactivo. ISBN 970-694-114-2.

Inayatullah, M., Shaw, S. R., Quicke, D. L. J. (1998) The genus Vipio Latreille (Hymenoptera: Braconidae) of America North of Mexico. Journal of Natural History, 32, 117-148. https://doi.org/10.1080/00222939800770071

INEGI. Instituto Nacional de Estadística y Geografía (1996) Estudio Hidrológico del estado de Baja California Sur. Instituto Nacional de Estadística, Geografía e Informática, Aguascalientes, México. $206 \mathrm{pp}$.

LaSalle, J., Gauld, I. D. (1993) Hymenoptera and Biodiversity. CAB International. Universidad de Michigan, EUA. 348 pp.

McIntosh, C. (1855) The book of the garden Vol. 2. W. Blackwood and Sons. Universidad de Michigan, EUA. 853 pp.

Quicke, D. L. J. (2012) We know too little about parasitoid wasp distributions to draw any conclusions about latitudinal trends in species richness, body size and biology. PLoS ONE, 7, e32101.

Rodríguez, J. C., Flores-Dávila, M., Coronado-Blanco, J. M., García-Martínez, O., Aguirre-Uribe, L. A. (2017) Estudio preliminar de la familia Braconidae (Hymenoptera) en Aguascalientes. In: Bernal, J. S. (Ed). XL Congreso Nacional de Control Biológico. Mérida, Yucatán, México.

Sánchez-García, J. A., Wharton, R. A., Romero-Nápoles, J., González-Hernández, A. (2002) Revisión del género Blacus Nees para México (Hymenoptera: Braconidae). Pp. 511-519. In: RomeroNápoles, J., Estrada-Venegas, E. G., Equihua-Martínez, A. (Eds). Entomología Mexicana. Ed. Sagitario. Vol. I. México.

Sarukhán, J., Koleff, P., Carabias, J., Soberón, J., Dirzo, R., Llorente-Bousquets, J., Anta, S. (2009) Capital natural de México. Síntesis: conocimiento actual, evaluación y perspectivas de sustentabilidad. Comisión Nacional para el Conocimiento y Uso de la Biodiversidad. México, 100 pp.

Siqueiros-Delgado, M. E., Rodríguez-Avalos, J. A., Martínez-Ramírez, J., Sierra-Muñoz, J. C. (2016) Situación actual de la vegetación del estado de Aguascalientes, México. Botanical Sciences, 94, 455-470.

Tucker, E. M., Chapman, E. G., Sharkey, M. J. (2015) A revision of the New World species of Cremnops Förster (Hymenoptera: Braconidae: Agathidinae). Zootaxa, 3916, 1-83. http://dx.doi.org/10.11646/zootaxa.3916.1.1.

Villegas, Á., Angón, H. C., Coordinación, A. (2008) La biodiversidad en Aguascalientes: estudio de Estado.

Wharton, R. A., Marsh P. M., Sharkey M. J. (1998) Manual para los géneros de la familia Braconidae (Hymenoptera) del Nuevo Mundo. The International Society of Hymenopterists. Washington, D.C. $447 \mathrm{pp}$.

Yu, D. S., van Achterberg, C., Horstmann, K. (2004) Taxapad. Ichneumonoidea. Vancouver, Canadá. (URL: www.Taxapad.com).

Yu, D. S., van Achterberg, C., Horstmann, K. (2016) Taxapad 2016. Ichneumonoidea 2015 (Biological and taxonomical information), Taxapad Interactive Catalogue Database on flash-drive. Nepean, Ottawa.

Zhang, Z. Q. (2011) Animal biodiversity: An introduction to higher-level classification and taxonomic richness. Zootaxa, 3148, 7-12. 